\title{
An Indoor Training Bike to Provide Real-time Feedback on the Aerodynamic Cycling Position using Frontal Area Calculations
}

\author{
Thomas PEETERS ${ }^{* 1}$, Raman GARIMELLA ${ }^{1,2}$, Stijn VERWULGEN ${ }^{1}$ \\ ${ }^{1}$ Department Product Development, Faculty of Design Sciences, University of Antwerp, Belgium; \\ ${ }^{2}$ Voxdale, Belgium \\ https://doi.org/10.15221/20.24
}

\begin{abstract}
An incorrect position or movement or slight alteration in position during cycling can induce an increase of aerodynamic resistance, which has an immense influence on the performance. However, when defining an optimal aerodynamic position as in wind tunnel experiments, there is no control over the maintaining of this position during training or races. Furthermore, during well-defined training methods on indoor smart trainers, the aerodynamic effect has not been taken into account in determining the imposed resistance.

Therefore, an indoor training bike was developed to continuously calculate the projected frontal area of the cyclist and the bike as an indicator of aerodynamic drag to estimate the wind resistance the cyclist experience in that position. The power a cyclist in that pose must push is calculated and automatically loaded to a smart trainer. Additionally, real-time feedback on the most efficient and aerodynamic position is provided. Therefore, vibrotactile instructions are provided when cyclists exceed a certain calibrated projected frontal area value, corresponding to the most aerodynamic position.

In this study, an intervention with only changing resistance based on the aerodynamics was compared with interventions with additionally vibrotactile feedback. The addition of vibrotactile signals provides significantly higher accuracy in recapturing the most optimal aerodynamic position $(p<0.001)$ including both competitive cyclists and amateur cyclists, as well as experiments with a time trial bike and a road bike. The results in this study show that the system with variation of resistance depending on the aerodynamic position, in combination with vibrotactile feedback for alerting cyclists when deviating from their optimal aerodynamic position can be an interesting tool to use during training sessions on a smart trainer.
\end{abstract}

Keywords: aerodynamics, frontal area, cycling, vibrotactile feedback

\section{Introduction}

Many cycling training programs include indoor training sessions on smart trainers, as power training or interval training, since these systems allow to follow the predefined intervals more strictly and in a safer way than in outdoor situations. Furthermore, indoor cycling apps (e.g. Zwift, Zwift Inc) provide realistic environments to simulate real-life cycling experiences by adapting the resistance for e.g. climbing and riding in a peloton. However, the influence of the posture and movement of the cyclist is not included in estimating the imposed resistance, causing that the effect of the biomechanical movement and the aerodynamic drag is neglected.

These biomechanical and aerodynamic parameters, however, have an important impact on cycling races and should be given attention during training as well [1], [2]. Aerodynamics has the highest influence on cycling performance and is especially effective in time trials, but is also important in road races [3]. Aerodynamic drag is dependent on both the bike and cyclist, where the posture of the cyclist itself has the biggest influence and is the most difficult to control [4]-[6]. Wind tunnel tests, computer aided design (CAD) models and projected frontal area calculations are most used to estimate the aerodynamic efficiency of a cyclist [4]. Once the optimal aerodynamic position is defined using one of the above-mentioned methods, cyclists lack feedback on the quality of retaining that optimal pose during training or races.

However, projected frontal area calculations can be real-time provided in any room, inducing that combining these calculations with a smart training system enables adapting the resistance based on the aerodynamic drag. In this way, real-time drag data can be provided during indoor training sessions to compare several cycling poses in realistic cycling situations. Furthermore, when the optimal aerodynamic position is known and calibrated, real-time feedback on the quality of the cyclists' position can be provided.

\footnotetext{
* thomas.peeters2@uantwerpen.be
} 
In past studies, vibrotactile feedback was used in several applications to guide athletes [7]-[11]. Vibrotactile feedback is defined as guiding subjects to a desired reference position or movement by applying vibrational stimuli onto subjects' skin. The actual condition of subjects is compared to the predefined reference condition and subjects are alerted by vibrating elements when deviating from this reference position [12].

This study aimed to develop an indoor training bike to allow training of the aerodynamic cycling position in realistic circumstances. Therefore, the projected frontal area of the cyclist was used as an indicator of aerodynamic drag and as input to provide resistance depending on the pose of the cyclist. In addition, real-time vibrotactile feedback was applied to the most optimal aerodynamic position. In this study, the effectiveness of this method was investigated for time trial bikes and road bikes, as well as for amateur cyclists and competitive riders.

\section{Development indoor training bike}

The indoor training bike combines a frontal camera to calculate the projected frontal area of the cyclist with a smart trainer, where the resistance changes based on the calculated projected frontal area. Additionally, a vibrating element is used to provide real-time vibrotactile feedback on the quality of maintaining the optimal aerodynamic position.

\subsection{Frontal camera and smart trainer}

The camera is an Intel ${ }^{\circledR}$ RealSense ${ }^{\mathrm{TM}}$ depth camera D415, with a $1280 \times 720$ active stereo depth resolution, 1920x1080 RGB resolution, a depth diagonal field of view of $70^{\circ}$ and a frame rate of $90 \mathrm{fps}$ frame for high-quality video images during movement. The camera registers the frontal view of the subject and calculates the projected frontal area using pixel calculation and depth correction (Figure 1 Left). To provide reliable measurements in different locations and situations, a calibration procedure without bike and cyclist is required to eliminate noise and objects in the scope of the camera.

Based on the calculated projected frontal area, an indication of the wind resistance and simultaneously the power cyclists should push in that position is determined. The power ( $\mathrm{P}$ in Watt) is calculated using equation 1 and combines the effects of the drivetrain efficiency, the velocity $(v$ in $\mathrm{m} / \mathrm{s})$, the rolling resistance indicated by a coefficient and the mass of the bike and cyclist ( $\mathrm{m} \mathrm{in} \mathrm{kg}$ ), and the air resistance indicated by a coefficient, the projected frontal area (PFA in $\mathrm{m}^{2}$ ) and the velocity [13]-[15].

$$
P=1.053 * v *\left(0.03 * m+0.615 * P F A * v^{2}\right)
$$

The power is constantly imposed on the smart trainer (Wahoo KICKR, Wahoo Fitness, Figure 1 Right) to provide a realistic cycling experience depending on the position of the cyclist. As such, the variation in air resistance by changing positions is automatically adapted and can be directly felt by the cyclist. Furthermore, an application was developed to visualize live data of the projected frontal area, power and velocity (Figure 2) to enable real-time interpretation of the effect of different poses for both cyclists and trainers. The application also allows us to define an optimal projected frontal area corresponding to the most aerodynamic cycling position, which can be used as reference position to compare and analyse the effect of different poses.

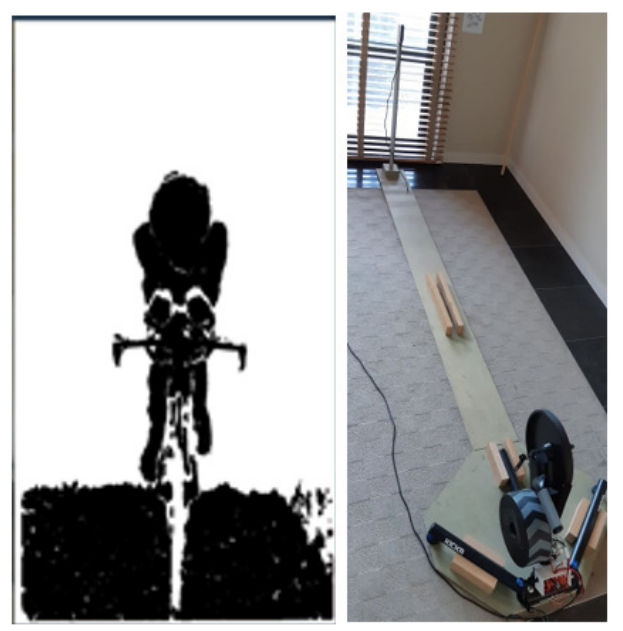

Fig. 1. The indoor training bike. Left: An image from the frontal camera to calculate the projected frontal area based on pixel calculation and depth correction. Right: the indoor training bike setup consisting of the frontal camera and a smart trainer to simulate resistance based on the frontal area calculations. 


\subsection{Vibrotactile feedback}

To provide feedback on the defined optimal aerodynamic cycling position, vibrotactile guidance is used when cyclists deviate from their optimal position. A certain margin of error above the optimal projected frontal area is allowed to eliminate the effect of noise and to avoid annoying the subjects by continuously providing vibrotactile feedback. A vibrating element (Vibration Motor, SparkFun Electronics) is attached to the processus spinosus C7 [16] (Figure 3 ) and is activated wirelessly using an Arduino Feather 32u4 Bluefruit LE and HC-12 module when cyclists exceed the margin of error.

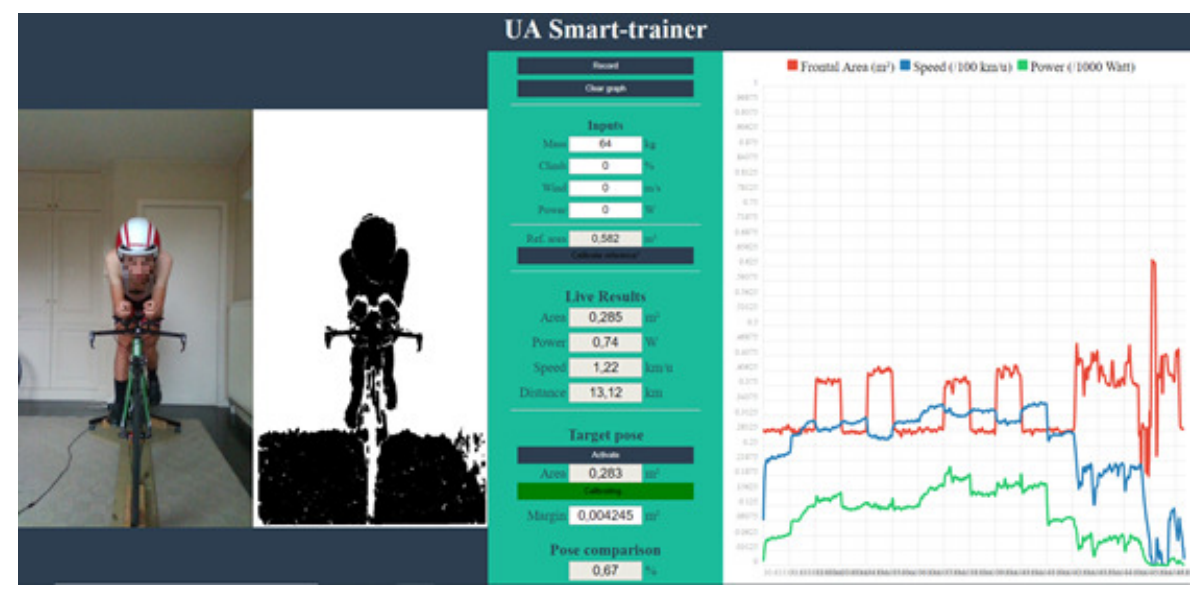

Fig. 2. The application to provide live results of the projected frontal area, power and velocity. Furthermore, a target pose with minimal projected frontal area in a comfortable cycling position can be defined to enable real-time vibrotactile feedback when deviating from the optimal position.
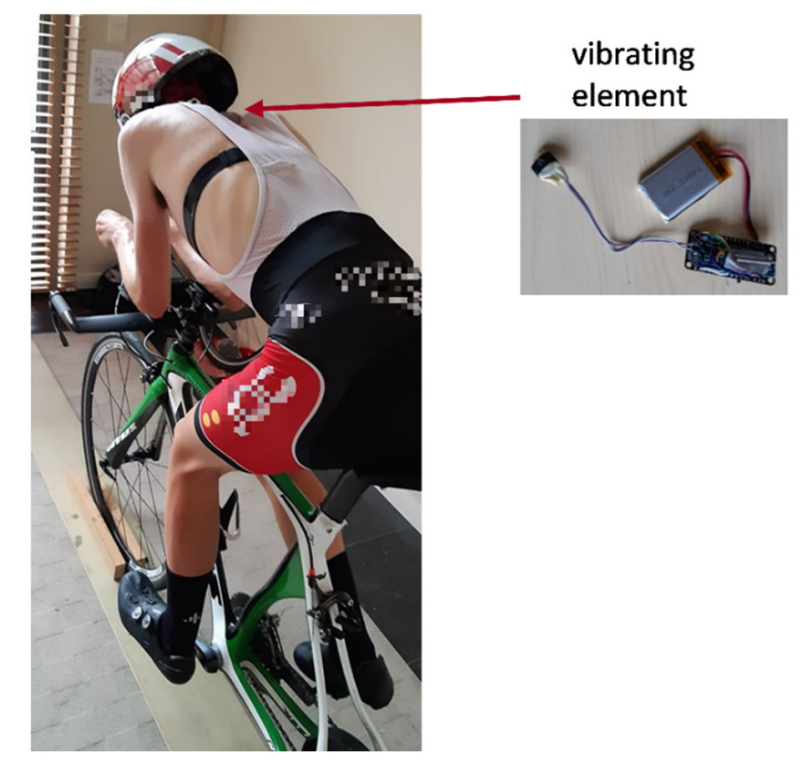

Fig. 3. The vibrating element, which is attached to processus spinosus C7, to provide vibrotactile feedback on the predefined most aerodynamic cycling position.

\section{Experiments}

Using the indoor training bike, the effect of changing resistance and vibrotactile feedback on (re)capturing the optimal aerodynamic cycling position was investigated. The main goal of this study was to investigate the effectiveness of the system for different bike types (time trial bike and road bike) and different levels of cyclists (amateurs and competitive riders).

\subsection{Procedure}

In this study, 14 participants were included consisting of seven amateur cyclists (average training hours per week of $5.9 \pm 3.0$ ) and seven competitive cyclists (average training hours per week of $13.4 \pm 6.1$ ). Six participants used a road bike during the experiments, eight used a time trial bike. The participants were instructed to follow a 12-minutes long protocol, where periods of one minute in the optimal 
aerodynamic position were altered with sitting upright as for taking a turn and coming out of the saddle as for climbing to investigate the effect on recapturing the optimal position. The subjects were instructed to follow the protocol three times in random order, where 1) only the resistance changed based on their pose, 2) additionally vibrotactile feedback with a margin of error of $1.5 \%$ was applied and 3 ) vibrotactile feedback with a margin of error of $3 \%$ was provided. During the vibrotactile feedback interventions, vibrations were only applied during the reference periods in the most aerodynamic position.

\subsection{Statistical analysis}

To allow in-between comparisons, the relative projected frontal area was calculated for each period in the most aerodynamic pose compared to the calibrated optimal aerodynamic position. The effect of vibrotactile feedback with different margins of error compared to only changing resistance, as well as interaction effects of competitiveness (competitive or amateur cyclist) and bike type (time trial bike or road bike) on the differences in relative projected frontal area between the three interventions was analysed using the repeated measures ANOVA test combined with possibly Bonferroni post-hoc analysis and between-subjects factors. The Independent T-test compared the differences in relative projected frontal area between competitive and amateur cyclists and between using a time trial bike and a road bike.

\section{Results}

The repeated measures ANOVA shows that the relative projected frontal area is significantly higher for the intervention with only resistance changing $(2.59 \pm 3.29 \%)$, compared to interventions with vibrotactile feedback $(-0.46 \pm 1.76 \%$ for the $1.5 \%$ margin of error, $-0.01 \pm 2.01 \%$ for the $3 \%$ margin of error) $(p<0.001)$. The competitiveness $(p<0.05)$ and bike type $(p<0.001)$ have an interaction effect on the recapturing of the reference position between the three interventions as shown in Figure 4 and Figure 5.

The Independent T-Test indicates no significant difference in attaining the aerodynamic reference position for competitive cyclists compared to amateur cyclists (competitive cyclists $0.57 \pm 2.99 \%$, amateur cyclists $0.76 \pm 2.49 \%, p=0.53$ ). The relative reference projected frontal area is significantly higher for participants with a road bike compared to riders with a time trial bike (road bike $1.04 \pm 3.44 \%$, time trial bike $0.37 \pm 2.04 \%, p<0.05)$.

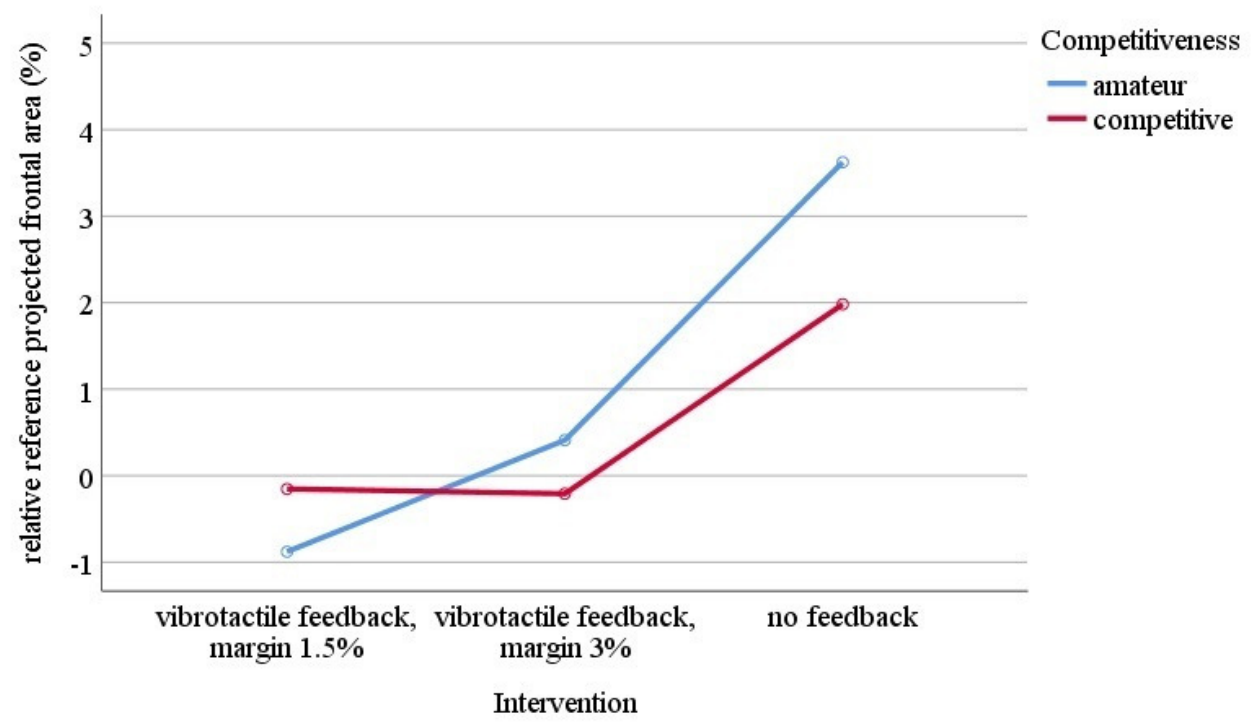

Fig. 4. The difference in relative projected frontal area between amateur and competitive cyclist for the three interventions. 


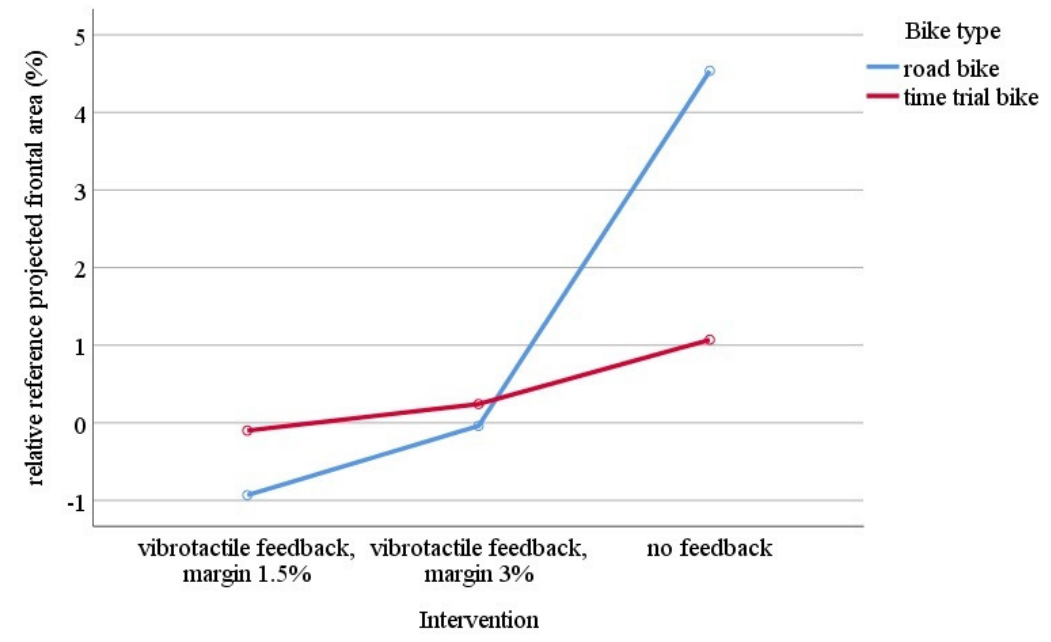

Fig. 5. The effect of bike type on the differences in relative projected frontal area for the three interventions

\section{Discussion}

This study demonstrates that the indoor training bike can be used to help cyclists to try and compare several poses during cycling on a smart trainer and to feel the real-life effect of change in resistance for each pose. The results show that vibrotactile feedback to remind cyclists of their most optimal aerodynamic cycling position provides higher accuracy in recapturing that pose compared to only using changing resistance.

For the comparison between the effect on the time trial bike and road bike, it is obvious that the absolute projected frontal area is higher for the road bike and that recapturing the reference cycling position using a time trial bike is easier. The time trial bike and especially the handlebar is engineered to fix and maintain cyclists in their most aerodynamic position [3], which limits the movement of the torso and arms during cycling effort to optimize the cycling performance [17]. The biggest difference in recapturing the reference position between time trial bike and road bike is found in the intervention with only changing resistance as shown in Figure 5 , which proves that vibrotactile feedback also can have utility during road races in specific circumstances such as in a breakaway [3].

Furthermore, there is no significant difference in relative projected frontal area between competitive and amateur cyclists. However, the influence of competitiveness on the relative projected frontal area is significantly different between the three interventions. For vibrotactile feedback with a margin of $1.5 \%$, the relative reference projected frontal area is lower and negative for amateur cyclists compared to competitive cyclists. This can be explained by the fact that competitive riders were already quite comfortable in the calibrated aerodynamic position and could better estimate if they recapture this position sufficiently accurately, where amateur cyclists tended to reach the position with the lowest possible projected frontal area as they received vibrotactile feedback. This argumentation is confirmed by the higher relative reference projected frontal area for amateur cyclists for the $3 \%$ margin and intervention with only changing resistance.

\section{Conclusion}

An indoor training bike was developed to automatically adapt the resistance on a smart trainer depending on the aerodynamic position of a cyclist. Furthermore, vibrotactile feedback was applied to alert cyclists when deviating from their optimal aerodynamic cycling position. The combination of changing resistance and vibrating signals has an added value in training the optimal aerodynamic position for both competitive and amateur cyclists and for training with a time trial bike and road bike. 


\section{References}

[1] M. Kordi, C. Fullerton, L. Passfield, and L. Parker Simpson, "Influence of upright versus time trial cycling position on determination of critical power and W' in trained cyclists," Eur. J. Sport Sci., vol. 19, no. 2, pp. 192-198, 2019, doi: 10.1080/17461391.2018.1495768.

[2] R. A. Lukes, S. B. Chin, and S. J. Haake, "The understanding and development of cycling aerodynamics," Sport. Eng., vol. 8, no. 2, pp. 59-74, 2005, doi: 10.1007/bf02844004.

[3] P. Mannion, Y. Toparlar, B. Blocken, E. Clifford, T. Andrianne, and M. Hajdukiewicz, "Aerodynamic drag in competitive tandem para-cycling: Road race versus time-trial positions," J. Wind Eng. Ind. Aerodyn., vol. 179, no. August 2018, pp. 92-101, 2018, doi: 10.1016/j.jweia.2018.05.011.

[4] P. Debraux, F. Grappe, A. V. Manolova, and W. Bertucci, "Aerodynamic drag in cycling: Methods of assessment," Sport. Biomech., vol. 10, no. 3, pp. 197-218, 2011, doi: 10.1080/14763141.2011.592209.

[5] N. Barry, D. Burton, J. Sheridan, M. Thompson, and N. A. T. Brown, "Aerodynamic performance and riding posture in road cycling and triathlon," Proc. Inst. Mech. Eng. Part P J. Sport. Eng. Technol., vol. 229, no. 1, pp. 28-38, 2015, doi: 10.1177/1754337114549876.

[6] P. F. J. Merkes, P. Menaspà, and C. R. Abbiss, "Reducing aerodynamic drag by adopting a novel road-cycling sprint position," Int. J. Sports Physiol. Perform., vol. 14, no. 6, pp. 733-738, 2019, doi: 10.1123/ijspp.2018-0560.

[7] K. Hasegawa and H. Shinoda, "Haptic Directional Instruction System for Sports," in Haptic Interaction, Lecture Notes in Electrical Engineering, vol. 432, 2018, pp. 361-368.

[8] A. J. Jansen, M. Dekker, and D. van der Steen, "The Vibe of Skating; Design and Testing of a Vibro-Tactile Feedback System," in 12th Conference of the International Sports Engineering Association, 2018, vol. 2, no. 6, p. 296, doi: 10.3390/proceedings2060296.

[9] L. Marchal-Crespo, M. Van Raai, G. Rauter, P. Wolf, and R. Riener, "The effect of haptic guidance and visual feedback on learning a complex tennis task," Exp. Brain Res., vol. 231, no. 3, pp. 277291, 2013, doi: 10.1007/s00221-013-3690-2.

[10] R. Sigrist, G. Rauter, R. Riener, and P. Wolf, "Augmented visual, auditory, haptic, and multimodal feedback in motor learning: A review," Psychon. Bull. Rev., vol. 20, no. 1, pp. 21-53, 2013, doi: 10.3758/s13423-012-0333-8.

[11] D. Spelmezan, "An investigation into the use of tactile instructions in snowboarding," in Proceedings of the 14th international conference on Human-computer interaction with mobile devices and services, 2012, pp. 417-426, doi: 10.1145/2371574.2371639.

[12] E. Van Breda, S. Verwulgen, W. Saeys, K. Wuyts, T. Peeters, and S. Truijen, "Vibrotactile feedback as a tool to improve motor learning and sports performance: a systematic review," BMJ Open Sport Exerc. Med., vol. 3, no. 1, 2017, doi: 10.1136/bmjsem-2016- 000216.

[13] D. Meyer, G. Kloss, and V. Senner, "What is Slowing Me Down? Estimation of Rolling Resistances during Cycling," Procedia Eng., vol. 147, pp. 526-531, 2016, doi: 10.1016/j.proeng.2016.06.232.

[14] R. Pivit, "Drag Force Formulas," Radfahren, pp. 44-46, 1990.

[15] J. García-López, J. A. Rodríguez-Marroyo, C. E. Juneau, J. Peleteiro, A. C. Martínez, and J. G. Villa, "Reference values and improvement of aerodynamic drag in professional cyclists," J. Sports Sci., vol. 26, no. 3, pp. 277-286, 2008, doi: 10.1080/02640410701501697.

[16] T. Peeters et al., "Vibrotactile Feedback during Physical Exercise: Perception of Vibrotactile Cues in Cycling," Int. J. Sports Med., vol. 40, no. 6, 2019, doi: 10.1055/a-0854-2963.

[17] J. McDaniel, A. Subudhi, and J. C. Martin, "Torso stabilization reduces the metabolic cost of producing cycling power," Can. J. Appl. Physiol., vol. 30, no. 4, pp. 433-441, 2005, doi: 10.1139/h05-132. 